ARTICLE

\title{
Broadband infrared LEDs based on europium-to- terbium charge transfer luminescence
}

Jonas J. Joos (10) 1,2凶, David Van der Heggen (10) 1,2, Lisa I. D. J. Martin (1) 1,2, Lucia Amidani (1) 3,7, Philippe F. Smet (10 1,2, Zoila Barandiarán ${ }^{4,5,6}$ \& Luis Seijo $4,5,6$

Efficient broadband infrared (IR) light-emitting diodes (LEDs) are needed for emerging applications that exploit near-IR spectroscopy, ranging from hand-held electronics to medicine. Here we report broadband IR luminescence, cooperatively originating from $\mathrm{Eu}^{2+}$ and $\mathrm{Tb}^{3+}$ dopants in $\mathrm{CaS}$. This peculiar emission overlaps with the red $\mathrm{Eu}^{2+}$ emission, ranges up to $1200 \mathrm{~nm}$ (full-width-at-half-maximum of $195 \mathrm{~nm}$ ) and is efficiently excited with visible light. Experimental evidence for metal-to-metal charge transfer (MMCT) luminescence is collected, comprising data from luminescence spectroscopy, microscopy and $\mathrm{X}$-ray spectroscopy. State-of-the-art multiconfigurational ab initio calculations attribute the IR emission to the radiative decay of a metastable MMCT state of a $\mathrm{Eu}^{2+}-\mathrm{Tb}^{3+}$ pair. The calculations explain why no MMCT emission is found in the similar compound SrS:Eu,Tb and are used to anticipate how to fine-tune the characteristics of the MMCT luminescence. Finally, a near-IR LED for versatile spectroscopic use is manufactured based on the MMCT emission.

\footnotetext{
${ }^{1}$ LumiLab, Department of Solid State Sciences, Ghent University, Krijgslaan 281/S1, 9000 Gent, Belgium. ${ }^{2}$ Center for Nano- and Biophotonics (NB Photonics), Ghent University, Technologiepark Zwijnaarde 15, 9052 Gent, Belgium. ${ }^{3}$ European Synchrotron Radiation Facility (ESRF), 71 Avenue des Martyrs, 38000 Grenoble, France. ${ }^{4}$ Departamento de Química, Facultad de Ciencias, Universidad Autónoma de Madrid, Módulo 13, c/Francisco Tomás y Valiente, Ciudad Universitaria de Cantoblanco, 28049 Madrid, Spain. ${ }^{5}$ Instituto Universitario de Ciencia de Materiales Nicolás Cabrera, Universidad Autónoma de Madrid, 28049 Madrid, Spain. ${ }^{6}$ Condensed Matter Physics Center (IFIMAC), Universidad Autónoma de Madrid, 28049 Madrid, Spain. ${ }^{7}$ Present address: Helmholtz-Zentrum Dresden-Rossendorf, 01314 Dresden, Germany. ${ }^{凶}$ email: jonas.joos@UGent.be
} 
$\mathrm{T}$ here is an urgent technological demand for broadband infrared (IR) phosphors to build phosphor-converted IR light-emitting diodes (pc-LEDs) with a broad spectral output. Broadband near-IR LEDs are a promising energy-efficient alternative for incandescent lamps for applications that rely on near-IR spectroscopy ${ }^{1,2}$. A large market that is presently explored is IR LEDs for the use in smart devices (e.g., mobile phones) to analyze food: information about its freshness, caloric value, or allergen content can be derived from the built-in spectrometer and IR LED ${ }^{3}$. Proof of concepts are demonstrated, but current state-of-the-art materials do not reach the desired energy efficiency, mostly due to the low absorption strength of the parityforbidden $3 d-3 d$ transitions of the $\mathrm{Cr}^{3+}$ or $\mathrm{Mn}^{2+}$ ions that are used $^{4-10}$. Alternatively, near-IR parity-allowed $\mathrm{Eu}^{2+} 5 d-4 f$ emission has been recently proposed ${ }^{11}$. Higher external quantum efficiencies can thus be achieved; however, at the cost of a smaller IR bandwidth. Material technologies and design strategies to obtain broadband IR phosphors that have a high absorption strength in the visible are hence highly desired to get this technology off the ground.

Other applications follow from the transparency of biological tissue for near-IR light and are hence situated in medicine ${ }^{12}$. This comprises imaging without the use of radioisotopes ${ }^{13-15}$, analyzing tissue during biopsy or endoscopy ${ }^{16}$, and IR therapy ${ }^{17}$. Also here, activators such as $\mathrm{Cr}^{3+}$ or $\mathrm{Nd}^{3+}$ feature substandard low excitation efficiencies due to the forbidden character of the used $3 d-3 d$ or $4 f-4 f$ transitions ${ }^{13,15}$. Circumventing this limitation can be done by incorporating $\mathrm{Eu}^{2+}$ in a suitable host, where the allowed $5 d-4 f$ emission is shifted towards longer wavelengths. A promising host for $\mathrm{Eu}^{2+}$ is $\mathrm{CaS}$, where emission $\sim 650 \mathrm{~nm}$ was shown in nanoparticles ${ }^{18,19}$. Upon co-doping with $\mathrm{Dy}^{3+}$, trapping can be induced upon ex situ UV excitation, after which red emission can be obtained upon in situ IR stimulation. As a downside, the red $\mathrm{Eu}^{2+}$ emission in this compound lies only partly in the first optical window of human skin tissue ${ }^{12}$, and would hence benefit from a further shift to the IR.

Large efforts have already been undertaken to optimize IR luminescent materials for the above-mentioned applications, starting from the well-known luminescence transitions such as
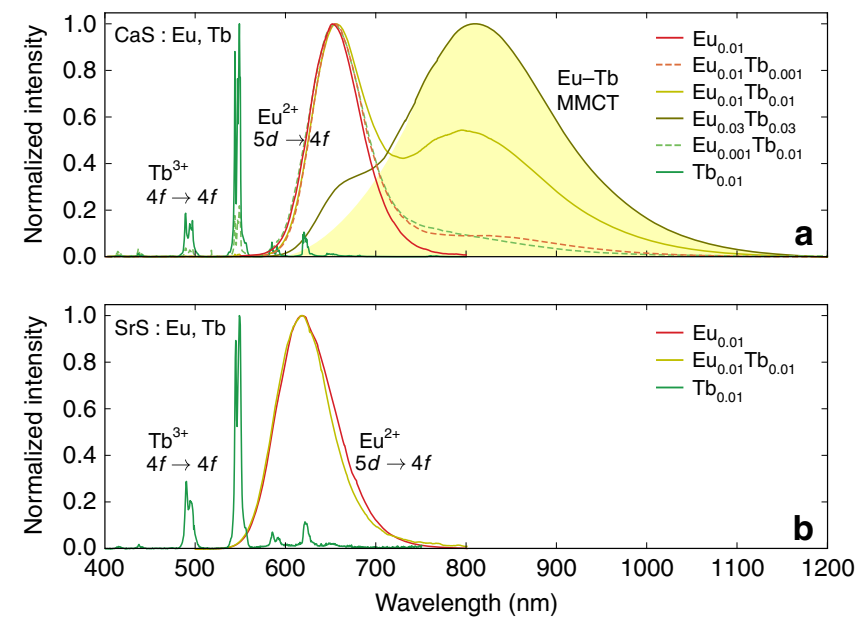

Fig. 1 Photoluminescence emission spectra. Photoluminescence emission spectra of $\mathrm{Eu}^{2+}$ - and $\mathrm{Tb}^{3+}$-codoped $\mathrm{CaS}(\mathbf{a})$ and $\mathrm{SrS}(\mathbf{b})$ phosphors. The spectra were collected upon $285 \mathrm{~nm}$ excitation for the Eu- and Eu/Tbcodoped powders and upon $305 \mathrm{~nm}$ excitation for the Tb-doped powders. The spectral shape of the infrared metal-to-metal charge transfer (MMCT) emission is highlighted in yellow as a guide to the eye. All spectra were measured at room temperature.
$3 d-3 d, 4 f-4 f$, or $5 d-4 f^{4-10,13,20}$. On the contrary, charge transfer (CT) states have remained below the radar because they often cause luminescence quenching, rather than generating luminescence themselves. Nonetheless, the so-called anomalous emission in several phosphors has recently been attributed to intervalence CT (IVCT) transitions ${ }^{21-26}$, that is, electron transfers between two lanthanide dopants that differ only in oxidation state. CT between two different lanthanide elements, that is, metal-to-metal CT (MMCT) states, has not been reported as such. The only exception is the tentative assignment of a direct lanthanideto-lanthanide $\mathrm{CT}$ absorption by Poolton et al. ${ }^{27}$ and concerns a $\mathrm{Ce}^{3+}+\mathrm{Sm}^{3+} \rightarrow \mathrm{Ce}^{4+}+\mathrm{Sm}^{2+} \mathrm{MMCT}$ in $\mathrm{YPO}_{4}$.

CT states between dopants remain often unnoticed because their absorption bands are very weak, yet they are important ${ }^{28}$. The multivalent nature of lanthanide and transition metal ions induces numerous $\mathrm{CT}$ states at low energy, intercalating with the excited states that are typically responsible for the luminescence of the individual ions. Therefore, they are expected to significantly alter the excited state dynamics by quenching existing or by generating new luminescent levels.

Here, broadband IR emission is reported upon addition of $\mathrm{Tb}$ to the red phosphor $\mathrm{CaS}: \mathrm{Eu}^{2+}$. This broadband IR emission can be efficiently pumped with long-wavelength visible light, substantiating an effective improvement of existing IR phosphors for pc-LED applications and for in vivo biomedicine without the need for prior ex situ charging nor expensive detection in the second or third optical window.

$\mathrm{CaS}: \mathrm{Eu}^{2+}, \mathrm{Tb}^{3+}$ and $\mathrm{SrS}: \mathrm{Eu}^{2+}, \mathrm{Tb}^{3+}$ are investigated in detail in a combined experimental-theoretical study that evidences that the observed IR emission is due to the radiative decay of MMCT states of $\mathrm{Eu}^{2+}-\mathrm{Tb}^{3+}$ pairs, a type of luminescence that was hitherto not described to the best of our knowledge. The ab initio multiconfigurational calculations explain why the MMCT luminescence is found for $\mathrm{CaS}: \mathrm{Eu}^{2+}, \mathrm{Tb}^{3+}$, but not for $\mathrm{SrS}: \mathrm{Eu}^{2+}, \mathrm{Tb}^{3+}$. This knowledge is utilized to show that MMCT emission can only be obtained when several conditions in terms of the electronic structure of the lanthanide pair, and the structural rigidity of the host crystal are fulfilled. This goes beyond the mere location of energy levels, but comprises vibrational frequencies and lanthanide-ligand bond lengths as well. The reported IR phosphor is finally applied to construct a broadband near-IR LED with a radiant output that surpasses the current state of the art.

\section{Results and discussion}

Photoluminescence spectra. The photoluminescence (PL) emission spectra for various $\mathrm{MS}: \mathrm{Eu}, \mathrm{Tb}(\mathrm{M}=\mathrm{Ca}, \mathrm{Sr})$ singly and codoped powders are shown in Fig. 1. Singly Eu-doped MS shows the characteristic broadband emission, peaking $\sim 650$ and $615 \mathrm{~nm}$ for CaS and SrS, respectively. This band is attributed to the radiative de-excitation of the $4 f^{6} 5 d^{1}$ states of $\mathrm{Eu}^{2+}$ towards the $4 f^{7}$ $\left({ }^{8} S\right)$ ground state ${ }^{29-31}$. The associated excitation spectrum as shown in Fig. 2 consists of a very broad band, ranging from 410 to $610 \mathrm{~nm}$, composed of numerous transitions towards the dense $4 f^{6} 5 d t_{2 g}^{1}$ manifold ${ }^{31,32}$. No trace of $\mathrm{Eu}^{3+}$ line emission is found. This does however not exclude its presence, because IVCT states are known to quench the $\mathrm{Eu}^{3+}$ emission in case it pairs with $\mathrm{Eu}^{2+}$ ions ${ }^{28}$. However, high-energy-resolution fluorescence-detected Xray absorption near-edge structure (HERFD-XANES ${ }^{33,34}$ ) spectra show that no $\mathrm{Eu}^{3+}$ is present in the prepared samples within the detection limits (see Supplementary Fig. 2).

Singly Tb-doped MS shows characteristic $\mathrm{Tb}^{3}+{ }^{5} D_{4} \rightarrow{ }^{7} F_{J}$ line emission across the visible range (green curve in Fig. 1), most notably in the green $(545 \mathrm{~nm}, J=5)$. This intraconfigurational $4 f^{8}$ emission can be excited by a relatively high-lying $4 f^{8} \rightarrow 4 f^{7} 5 d^{1}$ excitation band in the near-UV (Fig. 2) ${ }^{35-37}$. In case of $\mathrm{CaS}$ and 


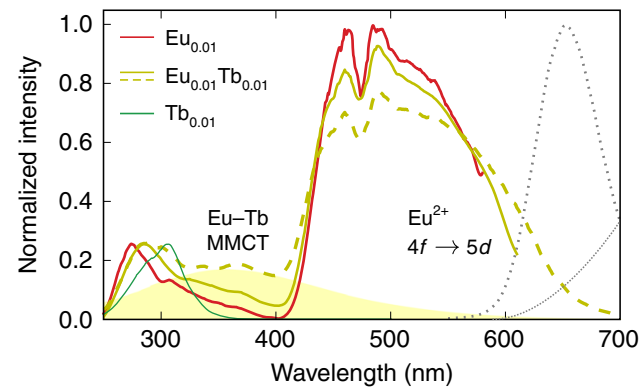

Fig. 2 Photoluminescence excitation spectra. Photoluminescence excitation spectra of $\mathrm{Eu}^{2+}$ - and $\mathrm{Tb}^{3+}$-codoped $\mathrm{CaS}$ phosphors. Spectra were collected for the $\mathrm{Tb}^{3+} 4 f-4 f$ emission ( $545 \mathrm{~nm}$, green solid lines), the $\mathrm{Eu}^{2+} 5 d-4 f$ emission ( $650 \mathrm{~nm}$, red and gold solid lines) and the Eu-Tb metal-to-metal charge transfer (MMCT) emission (790 nm, dashed line). The estimated spectral profile of the Eu-Tb MMCT excitation band is highlighted in yellow. The Eu ${ }^{2+} 5 d-4 f$ and Eu-Tb MMCT emission spectral profiles are shown in addition (gray dotted lines). All spectra were measured at room temperature.

SrS, the fundamental absorption of the host lies in the same energy range, and the near-UV absorption and excitation bands are likely the result of a mixture of host- and dopant-related transitions ${ }^{30,31}$.

When $\mathrm{Eu}$ and $\mathrm{Tb}$ are combined in the same sample, an additional IR emission band emerges in case of $\mathrm{CaS}$, but not in case of SrS. This band is highlighted in Fig. 1. It peaks at $810 \mathrm{~nm}$ $\left(12,345 \mathrm{~cm}^{-1}\right)$ and is very broad, with a full-width at halfmaximum of $195 \mathrm{~nm}\left(2960 \mathrm{~cm}^{-1}\right)$. On the high-energy side, it overlaps with the $\mathrm{Eu}^{2+} 4 f^{6} 5 d^{1} \rightarrow 4 f^{7}$ luminescence around $650 \mathrm{~nm}$ and extends up to $1100-1200 \mathrm{~nm}$. Its excitation spectrum (dashed curve in Fig. 2) is very similar as for the $\mathrm{Eu}^{2+}$ luminescence, where the $4 f^{7} \rightarrow 4 f^{6} 5 d t_{2 g}^{1}$ band can be identified, with a small redshift of $20 \mathrm{~nm}$, corresponding to about $500 \mathrm{~cm}^{-1}$. In addition to this band, some excitation intensity can be found in the region around $370 \mathrm{~nm}$ where no allowed transitions for $\mathrm{Eu}^{2+}$ are found ${ }^{31}$, suggesting the presence of additional excited states when $\mathrm{Eu}$ and $\mathrm{Tb}$ are codoped. These features are also visible at low temperature, as well as in the diffuse reflectance spectra (see Supplementary Figs. 6 and 7).

Lifetime measurements (see Supplementary Fig. 9 and Supplementary Table 1) indicate that the IR emission bands feature a very similar decay behavior than the $\mathrm{Eu}^{2+} 5 d \rightarrow 4 f$ emission, with time constants around $500 \mathrm{~ns}$ at room temperature ${ }^{38}$.

In order to exclude that this previously unseen emission band is due to the used precursors, codoped powders from different precursor batches were prepared, using fluorides, oxides, and sulfides as lanthanide precursors ${ }^{39-42}$. All syntheses where Eu and $\mathrm{Tb}$ were both present as dopants resulted in the same IR emission band, while this IR band was always absent in case that only one dopant was used (see Supplementary Fig. 4). The similarity of the IR emission regardless of the synthesis conditions suggests that the details of the charge compensation mechanism for $\mathrm{Tb}^{3+}$ do not directly affect the IR emission. Explicit compensation of $\mathrm{Tb}^{3+}$ by adding a monovalent codopant such as $\mathrm{Na}^{+}$is shown to be detrimental for the luminescence properties, leading to a efficiency drop of a factor 10 (see Supplementary Fig. 8). The reason is that, when $\mathrm{Tb}^{3+}$ is extrinsically compensated, the formation of $\mathrm{Eu}^{3+}$ will also be favored ${ }^{29}$, generating undesired $\mathrm{Eu}^{2+}-\mathrm{Eu}^{3+}\left(-\mathrm{Na}^{+}\right)$centers in addition to the intended $\mathrm{Eu}^{2+}-\mathrm{Tb}^{3+}\left(-\mathrm{Na}^{+}\right)$centers. The former centers are responsible for the luminescence efficiency drop by IVCT quenching 28,43 .
This check, along with the demonstrated phase purity (from Xray diffraction (XRD), see Supplementary Fig. 1) and the presence of only one oxidation state for both dopants in the absence of extrinsic charge compensation, that is, $\mathrm{Eu}^{2+}$ and $\mathrm{Tb}^{3+}$ (from HERFD-XANES, see Supplementary Fig. 2) strongly suggests that this IR emission band is a physical effect that emerges due to an interaction between the $\mathrm{Eu}^{2+}$ and $\mathrm{Tb}^{3+}$ centers in the calcium sulfide crystal.

To acquire more information about this peculiar luminescence, its properties are investigated as a function of the $\mathrm{Eu}$ and $\mathrm{Tb}$ doping concentrations. The PL emission spectra indicate that the $\mathrm{Tb}^{3+}$ intraconfigurational $4 f^{8}$ emission is firmly diminished upon the addition of $\mathrm{Eu}^{2+}$ ions until it completely vanishes (Fig. 1). This is not surprising due to the large overlap between the $\mathrm{Tb}^{3+}$ emission and $\mathrm{Eu}^{2+}$ excitation spectra, enabling an efficient energy transfer where the $\mathrm{Tb}^{3+}$ ion sensitizes the $\mathrm{Eu}^{2+}$ luminescence ${ }^{44,45}$. When a small amount of $\mathrm{Eu}^{2+}$ is added to a $\mathrm{Tb}^{3+}$-doped sample $\left(\mathrm{CaS}: \mathrm{Eu}_{0.001} \mathrm{~Tb}_{0.01}\right)$, or vice versa $(\mathrm{CaS}$ : $\left.\mathrm{Eu}_{0.01} \mathrm{~Tb}_{0.001}\right)$, the IR emission shows up, but with a limited intensity. The relative intensities are comparable in both cases (see Fig. 1). For higher concentrations, $1 \%$ for both dopants (CaS: $\mathrm{Eu}_{0.01}\left(\mathrm{~Tb}_{0.01}\right)$, the IR emission stands out, featuring a larger integrated intensity than the $\mathrm{Eu}^{2+} 5 d \rightarrow 4 f$ emission. Upon increasing the doping concentrations even more (CaS: $\left.\mathrm{Eu}_{0.03} \mathrm{~Tb}_{0.03}\right)$, the IR emission dominates the entire emission spectrum.

The emergence of the IR emission upon Tb addition to CaS:Eu is accompanied by a decrease in PL quantum efficiency (QE), which decreases from $35 \%$ for $\mathrm{CaS}^{\mathrm{E}} \mathrm{Eu}_{0.01}$ to $10 \%$ for $\mathrm{CaS}$ : $\mathrm{Eu}_{0.01} \mathrm{~Tb}_{0.01}$ (see Supplementary Fig. 8). This relatively low internal QE is partly compensated by the efficient excitability of the IR emission (see Fig. 2), where $>90 \%$ of the incident visible $(400-600 \mathrm{~m}$ ) light is absorbed (see Supplementary Fig. 7). It is hence clear that practical applications require a trade-off between conversion efficiency and the fraction of IR in the emission spectrum (see further) ${ }^{46}$.

Concentration dependence. The concentration-dependent PL study indicates that the intensity of the IR emission scales with the product of the concentrations of both dopants; however, drawing quantitative conclusions is hindered by the limited number of concentrations that can be prepared and does not account for uncontrollable microscopic concentration differences ${ }^{47-50}$. To get a more detailed picture, a microscopic study is performed.

For this, two grains with extreme doping inhomogeneity are explored $^{51-53}$. It should be stressed that these grains were selected for this purpose and that they do not represent the global doping homogeneity of the phosphors, which is much better. As shown in Supplementary Fig. 3 and the accompanying discussion, variations in local concentrations are limited to less than a percent, corresponding to a decent doping homogeneity.

The microscopic study of the inhomogeneous grains is shown in Fig. 3. One grain exhibits predominantly red emission (lower left), while the other grain shows a strong IR emission (upper right). From the elemental analysis by energy-dispersive X-ray spectroscopy (EDX), it is clear that the doping is indeed inhomogeneous and that the local $\mathrm{Eu}$ and $\mathrm{Tb}$ concentrations range from 0 to roughly $4 \%^{54}$. As a direct consequence, the cathodoluminescence $(\mathrm{CL})$ spectrum shows strong variations across the sample because the intensity of the IR emission depends strongly on the $\mathrm{Eu}$ and $\mathrm{Tb}$ concentrations. As an illustration, five local spectra are shown in Fig. 3c. It is clear that the smaller grain on the bottom of the image shows negligible IR emission (Fig. 3c, d), which is compatible with the elemental 

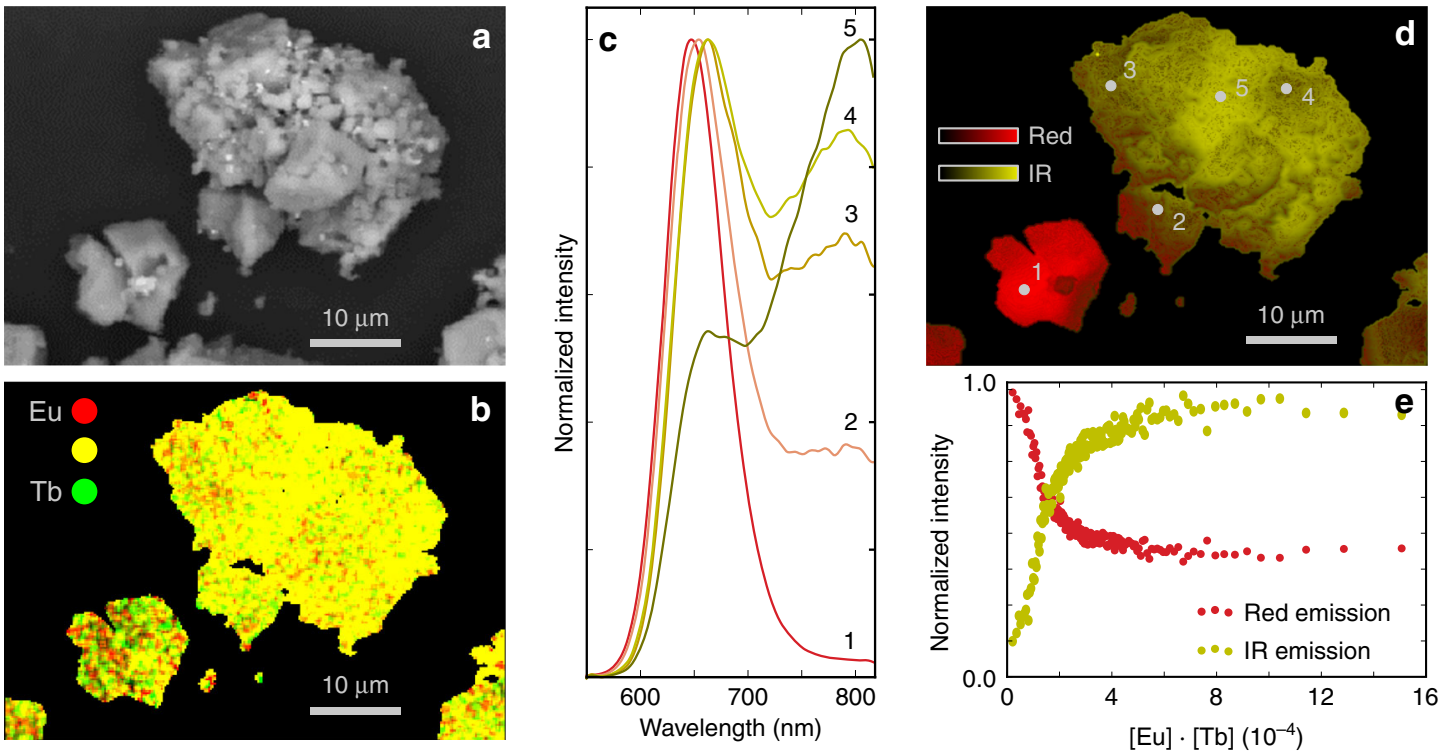

Fig. 3 Microscopic SEM-EDX-CL study. Scanning electron microscope (SEM) study on a CaS:Eu $\mathrm{E}_{0.01} \mathrm{~Tb}_{0.01}$ powder. Backscattered electron image (a), along with energy-dispersive X-ray (EDX) maps for terbium and europium (b). The colors encode the local terbium (green) and europium (red) concentrations. Simultaneous detection leads to a yellow color. c Cathodoluminescence $(\mathrm{CL})$ spectra for a few selected points that are indicated in d. d False color image, displaying the total integrated $\mathrm{CL}$ intensity. e shows how the relative contributions of the red $\mathrm{Eu}^{2+}$ (integrated from 600 to $650 \mathrm{~m}$ ) and IR (integrated from 760 to $815 \mathrm{~m}$ ) luminescence band to the total $\mathrm{CL}$ spectrum evolve as a function of the local Eu and Tb concentrations, [Eu] $\cdot[\mathrm{Tb}]$.

analysis that suggests that $\mathrm{Eu}$ and $\mathrm{Tb}$ are well separated in this grain, indicated by a limited amount of yellow in Fig. 3b. For the larger grain, $\mathrm{Eu}$ and $\mathrm{Tb}$ clearly congregate (there is more yellow in Fig. 3b) and intense IR emission is found (Fig. 3c, d).

From the PL study, it is clear that both $\mathrm{Eu}$ and $\mathrm{Tb}$ are required to induce IR emission; therefore, the product of both local concentrations, $[\mathrm{Eu}] \cdot[\mathrm{Tb}]$, determined per pixel in the scanning electron microscope (SEM)-EDX map, is used as dependent variable to correlate the luminescence properties to the CL spectrum, measured for the same pixel ${ }^{49,55}$. The red and IR contributions to the local CL spectrum are integrated and subsequently analyzed as a function of $[\mathrm{Eu}] \cdot[\mathrm{Tb}]$. Figure $3 \mathrm{e}$ was obtained by averaging data points along the abscissa. At low $[\mathrm{Eu}] \cdot[\mathrm{Tb}]$ values, the IR emission increases linearly as a function of $[\mathrm{Eu}] \cdot[\mathrm{Tb}]$, while the red $\mathrm{Eu}^{2+}$ emission decreases accordingly. After the linear increase/decrease, the relative intensities stabilize and the spectrum does not change appreciably upon increasing the product of the doping concentrations above roughly $3 \times 10^{-4}$, which corresponds to a symmetric doping concentration, $\sqrt{[\mathrm{Eu}] \cdot[\mathrm{Tb}]}$, of $1.7 \%$. No spot is found where the red $\mathrm{Eu}^{2+}$ emission completely disappears, not in the area displayed in Fig. 3, nor in any other grain that was investigated, nor in the emission spectra of phosphors with doping concentrations $>5 \%$ (see Supplementary Fig. 5). This equilibrium between red and IR emissions implies that the IR emission is presumably not the result of an energy transfer from $\mathrm{Eu}^{2+}$ to another emitting center because it would then be expected for the $\mathrm{Eu}^{2+}$ emission to vanish completely if the concentrations are stretched sufficiently ${ }^{44,56}$. Yet, the $\mathrm{Eu}^{2+}$ absorption bands are clearly present in the excitation spectrum of the IR band. This means that the IR emission stems directly from a $\mathrm{Eu}^{2+}$-containing defect cluster.

Temperature dependence. Measurement of the PL intensities as a function of temperature, that is, a thermal quenching (TQ) experiment can reveal more information about the equilibrium between the red and IR emissions. The result is shown in Fig. 4 . The red $\mathrm{Eu}^{2+}$ emission follows a rather standard behavior, which

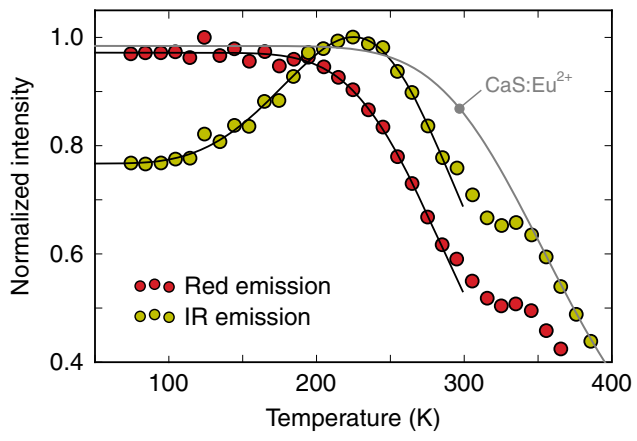

Fig. 4 Thermal quenching profiles. Thermal quenching curves for the red and infrared (IR) emission bands of $\mathrm{CaS}: \mathrm{Eu}_{0.01} \mathrm{~Tb}_{0.01}$, measured upon 470 $\mathrm{nm}$ excitation. The black curves are the result of fitting Eqs. (1) and (2) for the red and IR emissions, respectively. The small shoulder $\sim 340 \mathrm{~K}$ is likely due to thermoluminescence ( $T L$ ) and is not explored further here. The gray curve shows the thermal quenching of singly doped $\mathrm{CaS}: \mathrm{Eu}_{0.01}$ for comparison.

resembles the shape of a single-barrier model ${ }^{57}$,

$$
I_{\text {red }}(T)=\frac{I_{0}}{1+A \exp \frac{-\Delta E_{T, \text { red }}}{k_{\mathrm{B}} T}} .
$$

Using this phenomenological model to fit the data yields a barrier height of $\Delta E_{T, \text { red }}=1484 \mathrm{~cm}^{-1}\left(A=1.07 \times 10^{3}\right)$. This TQ performance is comparable to the one of singly doped $\mathrm{CaS}: \mathrm{Eu}_{0.01}$ phosphors, which contain sufficient $\mathrm{Eu}$ for concentration quenching to be noticeable $29,58,59$.

In contrast to the red emission, which shows the expected TQ behavior, the IR emission looks more complicated as a function of temperature, with an increase in intensity between 100 and $225 \mathrm{~K}$. This indicates that the IR emission is to some extent thermally activated. However, a substantial fraction of the IR emission, $\sim 77 \%$ of the maximal output at $225 \mathrm{~K}$, is also being emitted at low temperature. This is reminiscent of the temperature dependence of internal conversion (IC) and inter-system crossing (ISC) in 


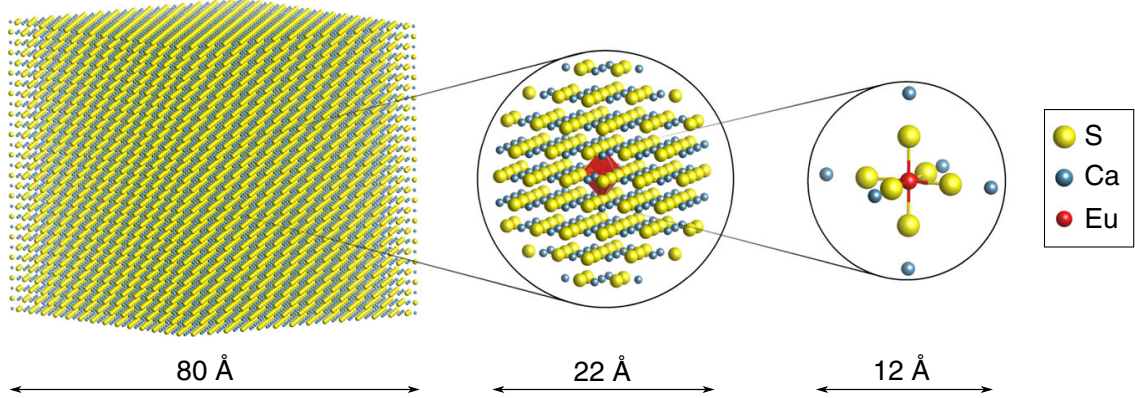

Fig. 5 Embedded cluster calculations. Graphical representation of the simulated system and how it is divided in the $\left(\mathrm{LnS}_{6} \mathrm{M}_{6}\right)^{2+/ 3+}(\mathrm{M}=\mathrm{Ca}, \mathrm{Sr}$ and $\mathrm{Ln}=$ Eu, Tb) cluster, which is treated at the highest level of theory (right), subject to the embedding potential composed of the full-ion ab initio model potentials (AIMP) of the 316 ions in the next four coordination shells (middle) and 24,389 point charges at the crystal lattice sites (left). The indicated distances are for $\mathrm{CaS}$ and are slightly larger for SrS.

\begin{tabular}{|c|c|c|}
\hline & CaS & SrS \\
\hline \multicolumn{3}{|l|}{ Eu-S equilibrium distances } \\
\hline $\mathrm{Eu}^{2+}\left({ }^{8} \mathrm{~S}_{7 / 2}\right)$ & 2.876 & 2.974 \\
\hline $\mathrm{Eu}^{3+}\left(1 A_{1 g}\right)$ & 2.729 & 2.800 \\
\hline Difference & 0.147 & 0.174 \\
\hline \multicolumn{3}{|l|}{ Tb-S equilibrium distances } \\
\hline $\mathrm{Tb}^{3+}\left(1 A_{1 g}\right)$ & 2.708 & 2.780 \\
\hline $\mathrm{Tb}^{2}+\left(1 \Gamma_{7 g / u}\right)$ & 2.833 & 2.948 \\
\hline Difference & -0.126 & -0.169 \\
\hline \multicolumn{3}{|l|}{$\mathrm{LnS}_{6}$ breathing mode } \\
\hline \multicolumn{3}{|l|}{ Vibrational frequencies } \\
\hline $\mathrm{Eu}^{2+}\left({ }^{8} \mathrm{~S}_{7 / 2}\right)$ & 286 & 273 \\
\hline $\mathrm{Tb}^{3+}\left(1 A_{1 g}\right)$ & 306 & 284 \\
\hline Mean values & 296 & 279 \\
\hline $\mathrm{Eu}^{3+}\left(1 A_{1 g}\right)$ & 307 & 286 \\
\hline $\mathrm{Tb}^{2+}\left(1 \Gamma_{7 g / u}\right)$ & 277 & 233 \\
\hline Mean values & 292 & 242 \\
\hline \multicolumn{3}{|l|}{ MMCT properties } \\
\hline Equilibrium energy & 19,968 & 20,403 \\
\hline Equilibrium $Q_{\mathrm{et}}$ & 0.428 & 0.548 \\
\hline \multicolumn{3}{|l|}{$\begin{array}{l}\text { Diabatic energy barriers for } \\
\text { hopping }\end{array}$} \\
\hline \multicolumn{3}{|l|}{$\mathrm{Eu}^{2+} \mathrm{Tb}^{3+} \leftrightarrow \mathrm{Eu}^{3+} \mathrm{Tb}^{2+}$} \\
\hline $1 \Gamma_{8 g} 1 A_{1 g} \rightarrow 1 A_{1 g} 1 \Gamma_{7 g / u}$ & 3406 & 3742 \\
\hline $1 \Gamma_{8 g} 1 A_{1 g} \leftarrow 1 A_{1 g} 1 \Gamma_{7 g / u}$ & 748 & 1602 \\
\hline \multicolumn{3}{|l|}{$\begin{array}{l}\text { Diabatic energy barriers for Non- } \\
\text { radiative decay MMCT }\end{array}$} \\
\hline $\mathrm{Eu}^{3+} \mathrm{Tb}^{2+} \leftrightarrow \mathrm{Eu}^{2+} \mathrm{Tb}^{3+}$ & & \\
\hline $1 A_{1 g} 1 \Gamma_{7 g / u} \rightarrow{ }^{8} S_{7 / 2} 3 A_{1 g}$ & 786 & 94 \\
\hline
\end{tabular}

Vertical electronic transition energies, diabatic energy barriers, and distance from the configurational coordinate diagrams. $\Gamma_{7 g}$ and $\Gamma_{7 u}$ are the lowest levels of $\mathrm{Tb}^{2+}$ in $\mathrm{CaS}$ and $\mathrm{SrS}$, respectively. Energies and vibrational frequencies in $\mathrm{cm}^{-1}$; distances in $\AA$.

molecular chromophores where rate constants are typically written as the sum of a temperature-dependent and temperature-independent term ${ }^{60,61}$. The IR TQ curve can hence be modeled by combining the ISC rate constant with a singlebarrier model for the TQ behavior,

$$
I_{\mathrm{IR}}(T)=\frac{I_{1}+I_{2} \exp \frac{-\Delta E_{\mathrm{ISC}}}{k_{\mathrm{B}} T}}{1+A \exp \frac{-\Delta E_{T, \mathrm{IR}}}{k_{\mathrm{B}} T}},
$$

where $I_{1}$ and $I_{2}$ represent the temperature-independent and temperature-dependent rate constants, respectively. $\Delta E_{\mathrm{ISC}}$ is the associated barrier height for the latter. Fitting Eq. (2) to the TQ profile of the IR emission yields $\Delta E_{\mathrm{ISC}}=476 \mathrm{~cm}^{-1}$ and $\Delta E_{T, \mathrm{IR}}=1500 \mathrm{~cm}^{-1}$ as barriers $\left(I_{1} / I_{2}=9.5\right.$ and $\left.A=1.61 \times 10^{3}\right)$.
The TQ at higher temperature is roughly the same as for the red emission, indicated by the similar energy barriers for quenching.

The intensities of the red and IR emission of the Eu,Tbcodoped phosphor already decrease at lower temperature with respect to the red emission of singly Eu-doped CaS (see gray curve in Fig. 4), featuring $\Delta E_{T, \text { red }}=1989 \mathrm{~cm}^{-1}\left(A=2.04 \times 10^{3}\right.$, Eq. (1)). This indicates that the addition of $\mathrm{Tb}$ opens an additional non-radiative decay channel that is active around room temperature. At higher temperatures, the TQ of the singly Eu-doped and Eu,Tb-codoped phosphors coincide, reaching $50 \%$ of the initial intensity at $T_{0.5} \approx 375 \mathrm{~K}$. This value is in correspondence with prior studies ${ }^{58,59,62}$, even though values of $475 \mathrm{~K}$ have been reported for single crystals $^{58}$, suggesting that efficiency gains are still feasible by optimizing the synthesis.

As shown by a model calculation by Struck and Fonger and subsequent surveys of experimental literature by various authors, the physical meaning of the above-determined energy barriers is rather limited due to tunneling effects and the importance of the details of the electron-vibrational structure on the non-radiative transition probabilities ${ }^{63-66}$. For that reason, Eqs. (1) and (2) should be regarded as strictly empirical prescriptions.

Qualitative interpretation of the above analysis suggests that an excited $\mathrm{Eu}^{2+}$ ion in the $\mathrm{Eu}^{2+}, \mathrm{Tb}^{3+}$-codoped material has two radiative decay possibilities, the standard red luminescence, and the IR luminescence which is achieved after some kind of internal transition towards another energy level. If both emissions would originate from the same initial level and differ in final level, the temperature-induced intensification of the IR emission would not be expected.

MMCT model. The experimental findings suggest that MMCT states might be involved in the complicated luminescence of this material. Given the oxidation states of the dopants in this compound, $\mathrm{Eu}^{2+}$ and $\mathrm{Tb}^{3+}$, the most probable scenario to be investigated are the $\mathrm{Eu}^{3+}-\mathrm{Tb}^{2+}$ MMCT states, that is, those where an electron is transferred from $\mathrm{Eu}^{2+}$ to $\mathrm{Tb}^{3+}$. To this means, $\mathrm{ab}$ initio embedded cluster calculations are employed (see Fig. 5).

Diabatic potential energy surfaces and configurational diagrams of $\mathrm{Eu}^{2+}-\mathrm{Tb}^{3+}$ pairs are obtained from the results of independent embedded cluster calculations ${ }^{23,67}$. This approach has proven its reliability by explaining the anomalous emission of several $\mathrm{Ce}$ - and $\mathrm{Yb}$-doped phosphors ${ }^{23,25,26,68}$, predicting the existence of absorption bands due to IVCT states in Eu-doped phosphors $^{28}$ and by showing the role of MMCT states at quenching luminescent levels of $\operatorname{Pr}^{3+69}$.

The configurational coordinate diagrams along the breathing mode for $\mathrm{Eu}^{2+}, \mathrm{Tb}^{3+}, \mathrm{Eu}^{3+}$, and $\mathrm{Tb}^{2+}$ are the ingredients for the electron transfer diagrams that describe the Eu-to-Tb MMCT transitions according to the recipe in refs. ${ }^{22,23}$. Calculations are 
performed for CaS and SrS hosts. Details, intermediate and final results are collected in Supplementary Tables 2-7 and in Supplementary Figs. 10-15.

The excited state landscape of $\mathrm{Eu}^{2+}$ was previously discussed in detail in ref. ${ }^{31}$ and is practically defined by a single ground state level, $4 f^{7}\left({ }^{8} S_{7 / 2}\right)$, separated from a very dense $4 f^{6} 5 d^{1}$ manifold by $\sim 16,000-17,000 \mathrm{~cm}^{-1} . \mathrm{Eu}^{3+}$ and $\mathrm{Tb}^{3+}$ feature conjugate ground state configurations with $4 f^{6}\left({ }^{7} F_{0}\right)$ and $4 f^{8}\left({ }^{7} F_{6}\right)$ multiplets, respectively. The higher-lying $4 f^{6}\left({ }^{5} D_{0}\right)$ and $4 f^{8}\left({ }^{5} D_{4}\right)$ states are the main $4 f-4 f$ emitting levels. The $4 f^{N-1} 5 d^{1}$ configurations feature high excitation energies for these trivalent lanthanides and are hence unnecessary for the current calculation. For $\mathrm{Tb}^{2+}$, the $4 f^{8} 5 d^{1}$ and $4 f^{9}$ configurations are close in energy, the former constituting the ground state in $\mathrm{CaS}\left(1 \Gamma_{7 g}\right)$, while a reversed order is found for $\mathrm{SrS}\left(1 \Gamma_{7 u}\right)$. More details about the electronic structures of $\mathrm{Tb}^{3+}$ and $\mathrm{Tb}^{2+}$ in $\mathrm{CaS}$ and $\mathrm{SrS}$ are given in the Supplementary Discussion, in particular regarding the relative energy of the $4 f^{8} 5 d^{1}$ and $4 f^{9}$ manifolds of $\mathrm{Tb}^{2+}$. The equilibrium $\mathrm{Eu}-\mathrm{S}$ and $\mathrm{Tb}-\mathrm{S}$ bond lengths and breathing mode vibrational frequencies are summarized in Table 1. Prior comparisons with experimental results for $\mathrm{Eu}^{2+}$ and $\mathrm{Eu}^{3+}$ proved an excellent quantitative agreement of $<300 \mathrm{~cm}^{-1}$ for excitation energies and at most a few \% for equilibrium bond lengths and vibrational frequencies ${ }^{31}$.

Within the diabatic approximation, the energy level scheme of a lanthanide pair can be constructed by combining all the levels of the individual ions, the resulting energy is given by addition of the individual energies with the Coulomb and exchange energies between the two lanthanides. The last two contributions are assumed to be state independent ${ }^{67}$. The resulting energy levels are uniquely labeled by combining both individual labels. As an example, the ground state of a $\mathrm{Eu}^{2+}-\mathrm{Tb}^{3+}$ pair is denoted as $4 f^{7}$ $\left({ }^{8} S_{7 / 2}\right)-4 f^{8}\left(1 A_{1 g}\right)$. Diabatic potential energy curves have proven their use by successfully explaining qualitative trends of CT processes upon chemical substitutions in host compounds $22,23,28,43$.

For every energy level of the Eu-Tb pair, a two-dimensional potential energy surface is obtained, spanned by the breathing modes of the $\mathrm{EuS}_{6}$ and $\mathrm{TbS}_{6}$ moieties. Every point in this twodimensional space hence corresponds to a unique pair of $\left(d_{\mathrm{Tb}-\mathrm{S}}\right.$, $\left.d_{\mathrm{Eu}-\mathrm{S}}\right)$. The equilibrium point of the $\mathrm{Eu}^{2+}-\mathrm{Tb}^{3+}$ states correspond to a relatively large $d_{\mathrm{Eu}-\mathrm{S}}$ value (divalent ion) and relatively small $d_{\mathrm{Tb}-\mathrm{S}}$ value (trivalent ion). The $\mathrm{Eu}^{2+}-\mathrm{Tb}^{3+} 4 f^{6} 5 d^{1}$ $\left(1 \Gamma_{8 g}\right)-4 f^{8}\left(1 A_{1 g}\right)$ potential energy surface is represented in the top panels of Fig. 6 for CaS and SrS (green contours), along with the lowest MMCT level, $\mathrm{Eu}^{3+}-\mathrm{Tb}^{2+} 4 f^{6}\left(1 A_{1 g}\right)-4 f^{8}\left(1 \Gamma_{7 \mathrm{~g}}\right)$ (black contours), where $d_{\mathrm{Eu}-\mathrm{S}}$ decreased (trivalent ion) and $d_{\mathrm{Tb}-\mathrm{S}}$ increased (divalent ion). The intersection of both potential energy surfaces is a curved line (dashed red line in the contour plots of Fig. 6).

The lower panels of Fig. 6 display the configurational coordinate diagrams for $\mathrm{Eu}^{2+}-\mathrm{Tb}^{3+}$ pairs in both host compounds along the electron transfer reaction coordinate, $Q_{\mathrm{et}}$. This coordinate is shown in the contour plots and is defined here as the piecewise straight line that connects the equilibria of the $\mathrm{Eu}^{2+}-\mathrm{Tb}^{3+} 4 f^{6} 5 d^{1}\left(1 \Gamma_{8 g}\right)-4 f^{8}\left(1 A_{1 g}\right)$ and $\mathrm{Eu}^{3+}-\mathrm{Tb}^{2+} 4 f^{6}\left(1 A_{1 g}\right)$ $-4 f^{8} 5 d^{1}\left(1 \Gamma_{7 \mathrm{~g}}\right)$ potential energy surfaces in the two-dimensional $\left(d_{\mathrm{Eu}-\mathrm{S}}, d_{\mathrm{Tb}-\mathrm{S}}\right)$ configurational space with their saddle point, that is, the minimum of the intersection of both surfaces. This onedimensional diagram is a simplification of the two-dimensional space that is probed for convenient visualization. Reported data such as location of minima, crossing points, barriers, and transition energies are however obtained from the twodimensional surfaces.

The configurational coordinate diagrams show that the $\mathrm{Eu}^{3+}-\mathrm{Tb}^{2+} \mathrm{MMCT}$ configuration gives rise to low-lying states, lying around $20,000 \mathrm{~cm}^{-1}$ above the ground state (Table 1$)$. The presence of these MMCT states alters the excited state dynamics after $4 f^{7} \rightarrow 4 f^{6} 5 d^{1}$ excitation of the $\mathrm{Eu}^{2+}$ ion. The excited electron can be non-radiatively transferred to the $\mathrm{Tb}^{3+}$ ion, forming a transient $\mathrm{Eu}^{3+}-\mathrm{Tb}^{2+}$ pair. Shortly, the $\mathrm{Tb}^{2+} 5 d$ electron is transferred back to the $\mathrm{Eu}^{3+} 4 f$ orbital, leading to a decay of the MMCT state.

Different decay channels are found in the case of $\mathrm{Eu}, \mathrm{Tb}$-codoped $\mathrm{CaS}$ or $\mathrm{SrS}$, evoked by their structural differences. In case of $\mathrm{CaS}$, the minimum of the lowest MMCT state $\left(4 f^{6}\left(1 A_{1 g}\right)-4 f^{8} 5 d^{1}\left(1 \Gamma_{7 g}\right)\right)$ is metastable. Therefore, radiative decay of this state can be expected to the structurally stressed $4 f^{7}\left({ }^{8} S_{7 / 2}\right)-4 f^{8}\left({ }^{6} F_{J}\right)$ ground state (red arrows in Fig. $6 \mathrm{c}$ ). In SrS, the minimum of the lowest MMCT state is crossed by the branches of the stressed $4 f^{7}\left({ }^{8} S_{7 / 2}\right)-4 f^{8}\left({ }^{6} F_{J}\right)$ states, enabling efficient non-radiative decay due to fast bottom crossover (red arrows in Fig. 6d) that impedes any radiative decay. The associated diabatic energy barrier is $94 \mathrm{~cm}^{-1}$, and will likely disappear or be of negligible size in an adiabatic calculation. This is indeed the behavior, which is experimentally found for $\mathrm{Eu}, \mathrm{Tb}$ codoped $\mathrm{CaS}$ and SrS.

Due to the large horizontal offset between ground and MMCT states, the resulting emission band in case of $\mathrm{CaS}: \mathrm{Eu}, \mathrm{Tb}$ is expected to be broad. The MMCT emission is predicted to start around $11,000 \mathrm{~cm}^{-1}(900 \mathrm{~nm})$, which is at slightly lower energy than the experimental IR emission, which starts $\sim 14,000-15,000 \mathrm{~cm}^{-1}$. This quantitative discrepancy between experimental and computed transition energies is in line with what can be expected from the diabatic approximation ${ }^{23,67}$.

The low-lying MMCT states do not only cause an additional emission band. Vertical excitation from the ground state towards the MMCT states is possible starting from about $28,000 \mathrm{~cm}^{-1}$ $(360 \mathrm{~nm})$, which coincides with the energy range where the lowspin $4 f^{6} 5 d t_{2 g}^{1}$ levels are found. The latter are spectroscopically invisible by direct excitation from the $4 f^{7}\left({ }^{8} S_{7 / 2}\right)$ ground state because of the spin selection rule ${ }^{31}$. The presence of the $\mathrm{Eu}-\mathrm{Tb}$ pairs and the MMCT states induces transition probability in this otherwise forbidden energy region as evidenced by the excitation band $\sim 370 \mathrm{~nm}$ in the experimental spectrum (Fig. 2). An estimated spectral shape is highlighted as a guide to the eye. This MMCT absorption is also visible in the excitation spectrum of the regular $\mathrm{Eu}^{2+}$ red emission at $650 \mathrm{~nm}$ for the $\mathrm{CaS}: \mathrm{Eu}_{0.01}$, $\mathrm{Tb}_{0.01}$ sample, indicating that a substantial fraction of the dopants can already interact in the studied concentration range.

The PL excitation spectrum of the MMCT emission (dashed line in Fig 2) indicates that it can be excited with the same wavelengths as the regular $\mathrm{Eu}^{2+}$ emission, even when the photon energy of the excitation light is insufficient to reach an MMCT branch by vertical excitation from the ground state. This can be explained by considering the double-well shape of the potential energy surface that is formed by the $\mathrm{Eu}^{2+}\left(1 \Gamma_{8 g}\right)-\mathrm{Tb}^{3+}\left(1 A_{1 g}\right)$ redemitting and the $\mathrm{Eu}^{3+}\left(1 A_{1 g}\right)-\mathrm{Tb}^{2+}\left(1 \Gamma_{7 \mathrm{~g}}\right)$ IR-emitting MMCT state. Even at low temperature, when the barrier cannot be thermally overcome, quantum mechanical tunneling will partially populate the MMCT state. This does not only explain why both emissions always appear together, but also why the IR emission is intensified when sufficient thermal energy is available to overcome the barrier and why the luminescent lifetimes for both emission bands are comparable.

Broadband IR LED. The broadband IR MMCT emission is now applied to construct an IR pc-LED that can directly be used for the numerous above-mentioned spectroscopic applications. Because of the high absorption strength of the parity-allowed $4 f-5 d$ transitions of $\mathrm{Eu}^{2+}$, higher external quantum efficiencies 

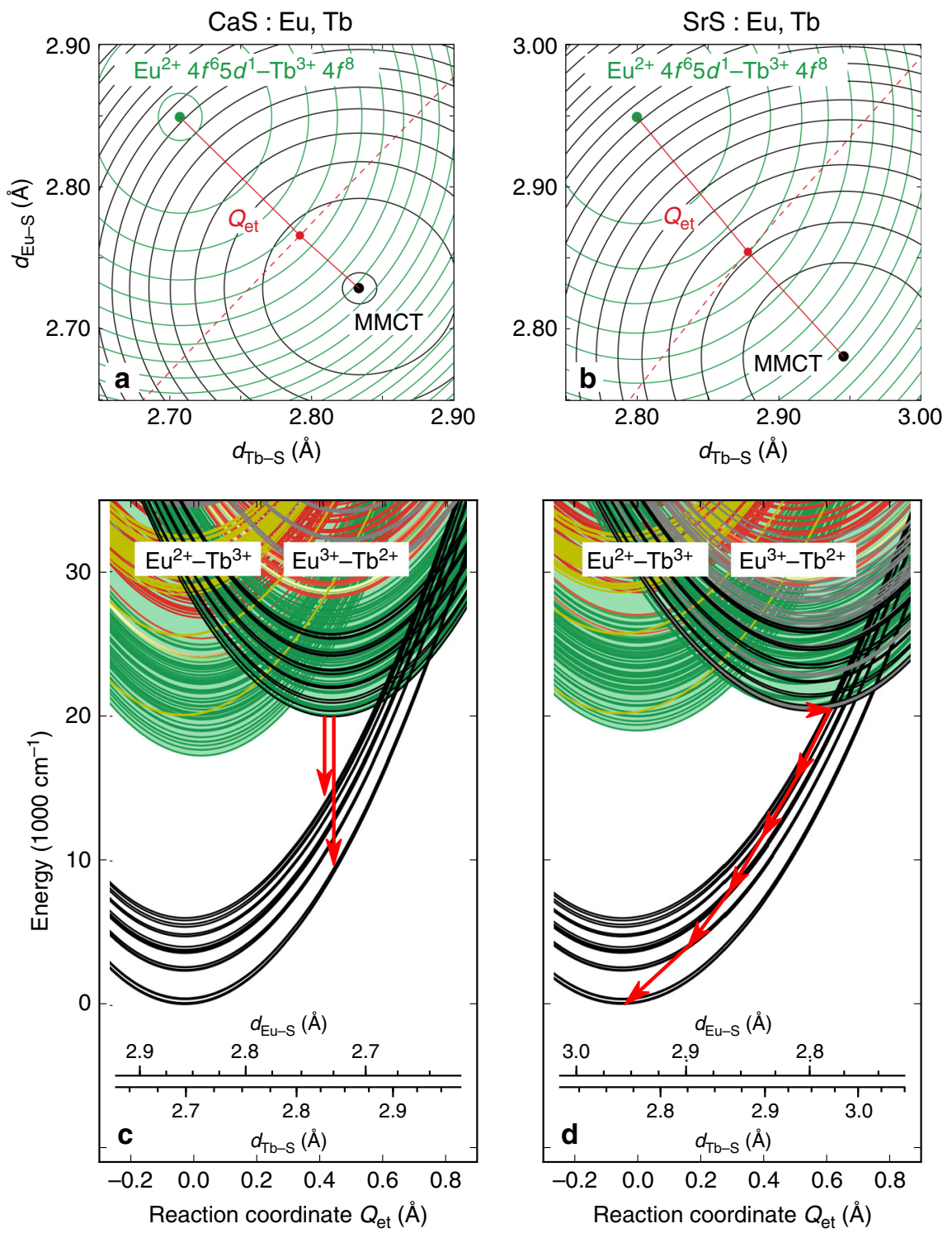

Fig. 6 Metal-to-metal charge transfer many-electron diagrams. Contour plots of selected potential energy surfaces in the two-dimensional configurational space $\left(d_{\mathrm{Eu}-\mathrm{S}}, d_{\mathrm{Tb}-\mathrm{S}}\right)(\mathbf{a}, \mathbf{b})$ and configurational coordinate diagrams along the electron transfer reaction coordinate $\left(Q_{\mathrm{et}}\right)(\mathbf{c}, \mathbf{d})$ for Eu-Tb

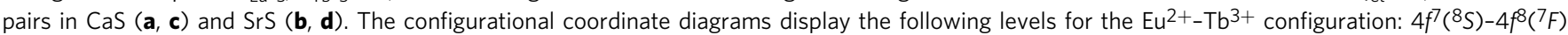
(black), $4 f^{6} 5 d t_{2 g}^{1}-4 f^{8}\left(1 A_{1 g}\right.$ ) (green for high-spin states, red for low-spin states) and $4 f^{7}\left({ }^{8} S\right)-4 f^{8}\left({ }^{5} D, L, G\right)$ (gold), and following levels for the Eu ${ }^{3+-T b^{2}+}$ configuration: $4 f^{6}\left({ }^{7} F\right)-4 f^{8} 5 d^{7}\left(1 \Gamma_{7 g}\right)$ (black), $4 f^{6}\left(1 A_{1 g}\right)-4 f^{8} 5 d t_{2 g}$ (green for high-spin states, red for low-spin states), and $4 f^{6}\left(1 A_{1 g}\right)-4 f^{9}$ (gray). The quasicontinuum formed by all other levels of the Eu-Tb pair, where none of both ions is in its ground state are represented by the green-colored background. The contour plots display the isolines corresponding to the $\mathrm{Eu}^{2+} 4 f^{6} 5 d^{1}\left(1 \Gamma_{8 g}\right)-\mathrm{Tb}^{3+} 4 f^{8}\left(1 A_{1 g}\right)$ and $\mathrm{Eu}^{3+} 4 f^{6}\left({ }^{7} F\right)-\mathrm{Tb}^{2}+4 f^{8} 5 d^{1}\left(1 \Gamma_{7 g}\right)$ (lowest metal-to-metal charge transfer (MMCT) state) surfaces. The reaction coordinate is indicated in red, along with the intersection between both surfaces (dashed red line).

can be achieved than with the current state-of-the-art, $\mathrm{Cr}^{3+}$-based phosphors ${ }^{4-6,8-10}$. Furthermore, the extremely broad MMCT emission extends the covered spectral range by several hundreds of nanometers to the IR compared to red/near-IR single $\mathrm{Eu}^{2+} 5 d-4 f$ emission $^{11}$.

Figure 7 displays the spectrum, expressed in $\mathrm{mW} \mathrm{nm}^{-1}$, for the obtained LED, where a blue $450 \mathrm{~nm}$ pumping LED was used. The LED features a width of $430 \mathrm{~nm}$ in the IR, ranging from 620 to $1050 \mathrm{~nm}$. The operation of the IR LED is illustrated by the pictures in Fig. 7b-e. Here, a high-pass cut-off filter of $780 \mathrm{~nm}$ is used to filter out the transmitted blue pumping light in order to appreciate the brightness of the IR emission. The pictures were taken with a camera that is also sensitive to near-IR light, as the emission is barely visible by the naked eye. The total radiant flux of the IR part of the emission amounts to $38 \mathrm{~mW}$. This value surpasses the current $\mathrm{Cr}^{3+}$-based state-of-the-art, having IR radiant fluxes in the range of $20-25 \mathrm{~mW}^{4-6,8-10}$, by $50 \%$.

It is clear that this broadband IR MMCT emission has a huge application potential. The next step comprises further optimization of its luminescence to arrive at higher efficiencies and to enable some spectral tuning to optimally meet the requirements of the different applications. As the luminescence mechanism in $\mathrm{CaS}: \mathrm{Eu}, \mathrm{Tb}$ was resolved in detail by our ab initio calculations, some prospects and further insights can be given based on these findings.

As shown, SrS is not a suitable host to obtain a luminescent MMCT state. There are two main differences between CaS and SrS that are crucial in this regard. First, the vibrational frequency is smaller by a few tens of $\mathrm{cm}^{-1}$ in case of $\operatorname{SrS}\left(242 \mathrm{~cm}^{-1}\right.$, compared to $292 \mathrm{~cm}^{-1}$ for CaS, see Table 1), which causes a slight 


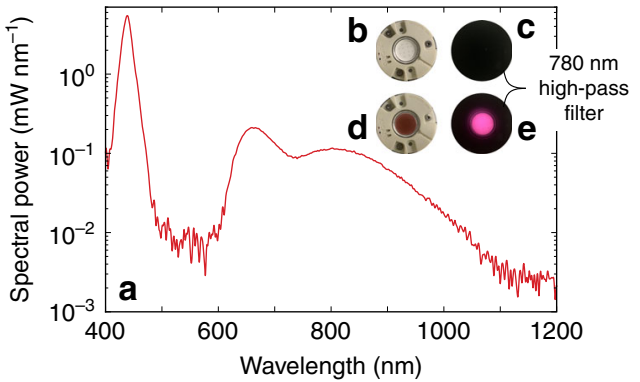

Fig. 7 Broadband near-IR LED. a Spectral output of the constructed broadband infrared (IR) light-emitting diode (LED). The inset shows the LED design, comprising a blue pumping LED (b), combined with the CaS:Eu, Tb broadband infrared phosphor (d), under operation below a 780-nm high-pass cut-off filter without (c) and with (e) the infrared phosphor present, captured by a camera with a near-IR responsivity.

opening of the branches that cross the MMCT state. This effect is not large because the vibrational frequency differs only by a small amount between $\mathrm{CaS}$ and SrS. Second, the offset in equilibrium geometry for the ground and MMCT states is larger for $\operatorname{SrS}\left(Q_{\mathrm{et}}=0.548 \AA\right.$, compared to $0.428 \AA$ for $\left.\mathrm{CaS}\right)$. This is the direct consequence of the larger bond length difference between divalent and trivalent lanthanides with respect to $\mathrm{CaS}$ (see Table 1) and causes the MMCT minimum to be pushed into the $4 f^{7}\left({ }^{8} S_{7 / 2}\right)-4 f^{8}\left({ }^{6} F_{J}\right)$ branches, causing quenching of the MMCT state. The latter parameter is the dominant one for the different behavior of $\mathrm{CaS}: \mathrm{Eu}, \mathrm{Tb}$ and $\mathrm{SrS}: \mathrm{Eu}, \mathrm{Tb}$.

The above analysis can be expanded to devise guidelines for finding other phosphors that exhibit MMCT luminescence. To achieve this, the ground state potential energy surface should cross the emitting MMCT level at a sufficiently large $Q_{\mathrm{et}}$ value. For this, small curvatures are required, which translates into small vibrational frequencies, a property that is not only typical for sulfide hosts ${ }^{30,70}$, but also for selenides $^{30}$, nitrides ${ }^{71}$, chlorides $^{72}$, bromides ${ }^{72}$, and iodides ${ }^{73}$. Additionally, the crossing between the ground state and the MMCT level can be shifted away from the MMCT minimum by decreasing the horizontal offset between both parabolas, that is, by decreasing the equilibrium $Q_{\text {et }}$ value. This value is proportional to the change in lanthanide-ligand bond length upon CT and to the square of the coordination number of the lanthanide ${ }^{67}$. MMCT luminescence will hence be more probable in hosts with a small site for the lanthanide as these experience smaller bond length changes. Ca-based hosts, preferably with a low coordination number, are hence more desirable with respect to $\mathrm{Sr}$ - or $\mathrm{Ba}$ based hosts.

When the chemical composition of host compounds is engineered to accomplish MMCT luminescence, also the vertical offset, and hence the MMCT emission energy, is expected to be affected. Indeed, the vertical offset is given by the difference of the ionization potential (IP) of the donor and the electron affinity (EA) of the acceptor, supplemented with the Coulomb and exchange interaction between both ions ${ }^{67}$, and these parameters are host dependent. A more radical manipulation of the vertical offset can be achieved by substituting the lanthanide ions. A rough idea on the effect of host modification and lanthanide substitution on the vertical offset can be acquired by consultation of the lengthy empirical data on IP's and EA's of lanthanide ions and their systematic behavior ${ }^{74,75}$.

In summary, the presence or absence of MMCT luminescence, and the emission energy is affected by three parameters: the local vibrational frequency, bond length change, and the selected lanthanide pair. A perfect balance between these parameters is required to achieve MMCT luminescence as in the case of $\mathrm{CaS}$ : $\mathrm{Eu}^{2+}, \mathrm{Tb}^{3+}$.

In this combined experimental-theoretical study, broadband IR emission in $\mathrm{CaS}: \mathrm{Eu}^{2+}, \mathrm{Tb}^{3+}$ is reported, characterized, and explained. The emission spectrum overlaps with the regular red $\mathrm{Eu}^{2+} 5 d-4 f$ luminescence and ranges up to $1200 \mathrm{~nm}$. Importantly, it can be efficiently pumped with long-wavelength visible light.

Concentration-dependent and microscopy experiments showed that the IR emission is caused by a cooperative effect between $\mathrm{Eu}$ and $\mathrm{Tb}$. $\mathrm{Ab}$ initio multiconfigurational calculations support that the IR-emitting state is a $\mathrm{Eu}^{3+}-\mathrm{Tb}^{2+}$ MMCT state whose local structure differs significantly from the $\mathrm{Eu}^{2+}-\mathrm{Tb}^{3+}$ ground state.

The type of host has a critical influence on the properties of the MMCT luminescence, as shown by the fact that the IR emission is quenched in the similar compound SrS. This behavior was explained by the ab initio calculations, which show that the location of MMCT states, and hence their luminescence properties can be fine-tuned by tweaking a few parameters. These are experimentally accessible by altering the anions and cations in the host, or the lanthanide pair, namely the local vibrational frequencies and structural rearrangement upon MMCT, as well as the IP and electron affinities of the dopants.

The CaS:Eu,Tb phosphor was used to construct a broadband IR pc-LED for spectroscopic applications in smart electronics, food safety, and medicine. The IR emission of the LED covers a 430-nm-wide spectral range in the red and near-IR. Moreover, this is achieved with an IR output radiant flux of $38 \mathrm{~mW}$, surpassing current state of the art.

\section{Methods}

Experimental method. Powders of $\mathrm{CaS}$ and $\mathrm{SrS}$, doped with $\mathrm{Eu}$ and/or $\mathrm{Tb}$ were prepared by a solid-state synthesis, using high-purity CaS (Alfa Aesar, 99.9\%), SrS (Alfa Aesar, 99.9\%), $\mathrm{EuF}_{3}$ (Alfa Aesar, 99.95\%), and $\mathrm{TbF}_{3}$ (Alfa Aesar, 99.9\%) as precursors. Stoichiometric quantities were weighed, mixed, and subsequently heat treated in a tube furnace for $2 \mathrm{~h}$ at $1000^{\circ} \mathrm{C}$ under a constant flow of $\mathrm{H}_{2} \mathrm{~S}$ gas. After the heat treatment, the samples were allowed to cool naturally. Finally, the samples were slightly ground and stored in an inert atmosphere. All samples were phase pure, as verified by powder XRD (see Supplementary Fig. 1). Doping concentrations that were reported are molar concentrations with respect to the cation, for example, CaS: $\mathrm{Eu}_{0.010} \mathrm{~Tb}_{0.001}$ is used for $\mathrm{Ca}_{0.989} \mathrm{Eu}_{0.010} \mathrm{~Tb}_{0.001} \mathrm{~S}$.

PL emission and excitation spectra were measured on an Edinburgh FS920, using a $450 \mathrm{~W}$ xenon arc lamp as excitation source and equipped with a Hamamatsu R928P red-sensitive photomultiplier (wavelength range from 200 to $850 \mathrm{~nm})$ and a Ge IR detector $(700-1600 \mathrm{~nm})$. Temperature-dependent PL was measured with the same spectrometer, equipped with a cryostat (Oxford Instruments Optistat CF).

The microscopy results were obtained with a Hitachi S-3400N SEM. A Thermo Scientific Noran System 7 EDX was used for chemical analysis and an optical fiber to collect the CL, which was subsequently analyzed by an Acton SP2300 monochromator and detected by a ProEM 1600 EMCCD (both Princeton Instruments). All shown spectra were properly calibrated for the spectral sensitivity of the various detectors.

An IR pc-LED was constructed using a blue pumping LED. For this a Xicato XTM LED module was used, operated at a constant current of $130 \mathrm{~mA}$, corresponding to a voltage of $16.7 \mathrm{~V}$. Its spectral radiant flux was obtained using a Thorlabs S401C thermal power meter.

Computational method. Diabatic potential energy surfaces and derived configurational coordinate diagrams were calculated for metal-to-metal electron transfer states of $\mathrm{Eu}^{2+} / \mathrm{Tb}^{3+}$ mixed valence pairs in $\mathrm{CaS}$ and $\mathrm{SrS}$, using the results of independent embedded cluster calculations as proposed in refs. ${ }^{22,23}$.

The electronic structures of the electron donor $(\mathrm{Eu})$ and acceptor $(\mathrm{Tb})$ octahedral embedded clusters $\left(\mathrm{LnS}_{6} \mathrm{M}_{6}\right)^{2+}$ and $\left(\mathrm{LnS}_{6} \mathrm{M}_{6}\right)^{3+}(\mathrm{M}=\mathrm{Ca}, \mathrm{Sr})$ were obtained with the suite of programs MOLCAS, ${ }^{76}$ using $D_{2 h}$ symmetry, in two-step spin-orbit coupling state-average restricted-active-space self-consistent-field (SARASSCF)/multi-state second-order perturbation theory (MS-RASPT2)/restrictedactive-space state-interaction spin-orbit (RASSI-SO) DKH calculations. In a first step, the spin-orbit-free many-electron relativistic second-order DKH

Hamiltonian ${ }^{77,78}$ was used to perform all-electron calculations using the same type of basis sets as in ref. ${ }^{31}$ : Gaussian atomic natural orbital relativistic basis sets ANORCC for $\mathrm{S}^{79}, \mathrm{Eu}$, and $\mathrm{Tb}^{80}$, with respective contractions $(17 s 12 p 5 d) /[6 s 5 p 3 d]$ 
(quadruple-zeta with polarization without $f$-functions quality) and

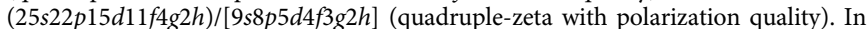
addition, the six-electron valence was explicitly added to the six alkaline earth metal ions next to the sulfur ligands in the [100] directions using adapted ANORCC basis sets, $\mathrm{Ca}(20 s 16 p 6 d) /[3 s 4 p 1 d]$ and $\operatorname{Sr}(23 s 19 p 12 d) /[3 s 4 p 1 d]$. The inner shells of these ions were frozen, using a $[\mathrm{Mg}]$ and $[\mathrm{Zn}]$ core for $\mathrm{Ca}$ and $\mathrm{Sr}$, respectively.

First, SA-RASSCF ${ }^{81-83}$ calculations were performed, allowing all possible occupations in the Ln $4 f$ shells and up to four electrons in the Ln $5 d, 6 s$, and $5 f$ shells, in order to account for the so-called double-shell effect ${ }^{84}$. Following states were obtained: those of the $4 f^{6}$ and $4 f^{8}$ configurations of $\mathrm{Eu}^{3+}$ and $\mathrm{Tb}^{3+}$ for which $2 S+1=7,5$, those of the $4 f^{7}, 4 f^{6} 5 d^{1}$, and $4 f^{8} 5 d^{1}$ of $\mathrm{Eu}^{2+}$ and $\mathrm{Tb}^{2+}$ for which $2 S+1=8,6$ and those of the $\mathrm{Tb}^{2+} 4 f^{9}$ configuration for which $2 S+1=6$. States with lower multiplicities were not considered because they are not expected to influence the lowest part of the energy spectrum that is of interest. Subsequently, MS-RASPT2 ${ }^{85-88}$ calculations allowed to correlate all cluster valence electrons, except the $4 d$ electrons of the lanthanides. A standard IPEA value $(0.25 \text { a.u. })^{89}$ and an imaginary shift of 0.15 a.u. $(\mathrm{Eu})$ or 0.50 a.u. $(\mathrm{Tb})$ was used. Second, the AMFI approximation of the $\mathrm{DKH}$ spin-orbit coupling operator was added to the Hamiltonian ${ }^{90}$ and RASSI-SO ${ }^{91,92}$ calculations were performed. Here, all states of a given cluster computed in the first step were allowed to interact.

In all calculations, the clusters were embedded in ab initio model potentials (AIMPs) ${ }^{93}$ that include Coulomb, exchange, and Pauli repulsion interactions from the $\mathrm{CaS}$ and SrS host lattices obtained in ref. ${ }^{31}$ from self-consistent embedded ions ${ }^{94}$ Hartree-Fock calculations ${ }^{76}$. Figure 5 shows how the small cluster is embedded in AIMPs and point charges.

\section{Data availability}

The data sets generated during and/or analyzed during the current study are available from the corresponding author on reasonable request.

\section{Code availability}

Multiconfigurational ab initio calculations were performed using MOLCAS.

Received: 29 October 2019; Accepted: 24 June 2020;

Published online: 20 July 2020

\section{References}

1. Tessler, N., Medvedev, V., Kazes, M., Kan, S. \& Banin, U. Efficient nearinfrared polymer nanocrystal light-emitting diodes. Science 295, 1506-1508 (2002).

2. Gong, X. et al. Highly efficient quantum dot near-infrared light-emitting diodes. Nat. Photonics 10, 253-257 (2016).

3. Halper, M. Osram's latest infrared LED identifies what's in your food (UPDATED). LEDs Magazine (2018).

4. Hayashi, D., van Dongen, A. M., Boerekamp, J., Spoor, S., Lucassen, G. \& Schleipen, J. A broadband LED source in visible to short-wave-infrared wavelengths for spectral tumor diagnostics. Appl. Phys. Lett. 110, 233701 (2017).

5. Zhang, L. et al. A high efficiency broad-band near-infrared $\mathrm{Ca}_{2} \mathrm{LuZr}_{2} \mathrm{Al}_{3} \mathrm{O}_{12}$ : $\mathrm{Cr}^{3+}$ garnet phosphor for blue led chips. J. Mater. Chem. C 6, 4967-4976 (2018).

6. Rajendran, V. et al. Super broadband near-infrared phosphors with high radiant flux as future light sources for spectroscopy applications. ACS Energy Lett. 3, 2679-2684 (2018).

7. Song, E. et al. Heavy $\mathrm{Mn}^{2+}$ doped $\mathrm{MgAl}_{2} \mathrm{O}_{4}$ phosphor for high-efficient nearinfrared light-emitting diode and the night-vision application. Adv. Opt. Mater. 7, 1901105 (2019).

8. Zeng, H., Zhou, T., Wang, L. \& Xie, R.-J. Two-site occupation for exploring ultra-broadband near-infrared phosphor-double-perovskite $\mathrm{La}_{2} \mathrm{MgZrO}_{6}: \mathrm{Cr}^{3+}$. Chem. Mater. 31, 5245-5253 (2019).

9. Wang, C. et al. An ultra-broadband near-infrared $\mathrm{Cr}^{3+}$-activated gallogermanate $\mathrm{Mg}_{3} \mathrm{Ga}_{2} \mathrm{GeO}_{8}$ phosphor as light sources for food analysis. ACS Appl. Electron. Mater. 1, 1046-1053 (2019).

10. Zhang, L. et al. $\mathrm{Cr}^{3+}$-doped broadband NIR garnet phosphor with enhanced luminescence and its application in NIR spectroscopy. Adv. Opt. Mater. 7, 1900185 (2019).

11. Qiao, J., Zhou, G., Zhou, Y., Zhang, Q. \& Xia, Z. Divalent europium-doped near-infrared-emitting phosphor for light-emitting diodes. Nat. Commun. 10, 5267 (2019).

12. Smith, A. M., Mancini, M. C. \& Nie, S. M. Second window for in vivo imaging. Nat. Nanotechnol. 4, 710-711 (2009).

13. Maldiney, T. et al. The in vivo activation of persistent nanophosphors for optical imaging of vascularization, tumours and grafted cells. Nat. Mater. 13, 418-426 (2014).
14. Eggebrecht, A. T. et al. Mapping distributed brain function and networks with diffuse optical tomography. Nat. Photonics 8, 448-454 (2014).

15. Xu, J. \& Tanabe, S. Persistent luminescence instead of phosphorescence: history, mechanism, and perspective. J. Lumin. 205, 581-620 (2019).

16. Evers, D. J. et al. Diffuse reflectance spectroscopy: a new guidance tool for improvement of biopsy procedures in lung malignancies. Clin. Lung Cancer 13, 424-431 (2012).

17. Ly, T. K. et al. Near-infrared organic light-emitting diodes with very high external quantum efficiency and radiance. Nat. Photonics 11, 63-68 (2017).

18. Rodriguez Burbano, D. C., Rodriguez, E. M., Dorenbos, P., Bettinelli, M. \& Capobianco, J. A. The near-IR photo-stimulated luminescence of CaS:Eu ${ }^{2}$ +/Dy ${ }^{3+}$ nanophosphors. J. Mater. Chem. C 2, 228-231 (2014).

19. Rodriguez Burbano, D. C., Sharma, S. K., Dorenbos, P., Viana, B. \& Capobianco, J. A. Persistent and photostimulated red emission in CaS:Eu ${ }^{2+}$ $\mathrm{Dy}^{3+}$ nanophosphors. Adv. Opt. Mater. 3, 551-557 (2015).

20. Xu, J., Murata, D., Ueda, J., Viana, B. \& Tanabe, S. Toward rechargeable persistent luminescence for the first and third biological windows via persistent energy transfer and electron trap redistribution. Inorg. Chem. 57, 5194-5203 (2018).

21. van Schaik, W., Lizzo, S., Smit, W. \& Blasse, G. Influence of impurities on the luminescence quantum efficiency of (La, Ce, Tb)PO $\mathrm{PO}_{4}$. J. Eur. Ceram. Soc. 140, 216-222 (1993).

22. Barandiarán, Z. \& Seijo, L. Intervalence charge transfer luminescence: interplay between anomalous and $5 \mathrm{~d}-4 \mathrm{f}$ emissions in $\mathrm{Yb}$-doped fluorite-type crystals. J. Chem. Phys. 141, 234704 (2014).

23. Seijo, L. \& Barandiarán, Z. Intervalence charge transfer luminescence: the anomalous luminescence of cerium-doped $\mathrm{Cs}_{2} \mathrm{LiLuCl}_{6}$ elpasolite. J. Chem. Phys. 141, 214706 (2014).

24. Kulesza, D., Cybińska, J., Seijo, L., Barandiarán, Z. \& Zych, E. Anomalous red and infrared luminescence of $\mathrm{Ce}^{3+}$ ions in SrS:Ce sintered ceramics. J. Phys. Chem. C 119, 27649-27656 (2015).

25. Strek, W. et al. Broadband anti-stokes white emission of $\mathrm{Sr}_{2} \mathrm{CeO}_{4}$ nanocrystals induced by laser irradiation. Phys. Chem. Chem. Phys. 18, 27921-27927 (2016)

26. Hughes-Currie, R. B. et al. X-ray excitation triggers ytterbium anomalous emission in $\mathrm{CaF}_{2}: \mathrm{Yb}$ but not in $\mathrm{SrF}_{2}: \mathrm{Yb}$. J. Phys. Chem. Lett. 8, 1175-1178 (2017).

27. Poolton, N. R. J., Bos, A. J. J., Jones, G. O. \& Dorenbos, P. Probing electron transfer processes in $\mathrm{YPO}_{4}: \mathrm{Ce}, \mathrm{Sm}$ by combined synchrotron-laser excitation spectroscopy. J. Phys. Condens. Matter 22, 185403 (2010).

28. Joos, J. J., Seijo, L. \& Barandiarán, Z. Direct evidence of intervalence chargetransfer states of Eu-doped luminescent materials. J. Phys. Chem. Lett. 10, 1581-1586 (2019).

29. Yamashita, N., Fukumoto, S., Ibuki, S. \& Ohnishi, H. Photoluminescence of $\mathrm{Eu}^{2+}$ and $\mathrm{Eu}^{3+}$ centers in CaS:Eu,Na phosphors. Jpn. J. Appl. Phys. 32, 3135-3139 (1993)

30. Yamashita, N., Harada, O. \& Nakamura, K. Photoluminescence spectra of Eu + centers in $\mathrm{Ca}(\mathrm{S}, \mathrm{Se}) \mathrm{Eu}$ and $\mathrm{Sr}(\mathrm{S}, \mathrm{Se}) \mathrm{Eu}$. Jpn. J. Appl. Phys. 34, 5539-5545 (1995).

31. Joos, J. J., Smet, P. F., Seijo, L. \& Barandiarán, Z. Insights into the complexity of the excited states of Eu-doped luminescent materials. Inorg. Chem. Front. 7, 871-888 (2020).

32. Weakliem, H. A. Electronic interactions in $4 \mathrm{f}^{6} 5 \mathrm{~d}$ configuration of $\mathrm{Eu}^{2+}$ in crystals. Phys. Rev. B 6, 2743-2748 (1972).

33. Gauthier, C., Sole, V. A., Signorato, R., Goulon, J. \& Moguiline, E. The ESRF Beamline ID26: X-ray absorption on ultra dilute sample. J. Synchrotron Radiat. 6, 164-166 (1999).

34. Glatzel, P. et al. Reflections on hard X-ray photon-in/photon-out spectroscopy for electronic structure studies. J. Electron Spectrosc. 188, 17-25 (2013).

35. Lehmann, W. Activators and co-activators in calcium sulfide phosphors. $J$. Lumin. 5, 87-107 (1972).

36. Tripathi, R. N. \& Sivaraman, S. Fluorescence spectra of CaS:(Tb, Eu) phosphors under X-ray excitation. Phys. Stat. Sol. A 55, K123-K126 (1979).

37. Leskelä, M., Niinistö, L., Nykänen, E., Soininen, P. \& Tiitta, M. Electroluminescent thin-films containing terbium-activated strontium sulfide. J. Less Common Met. 153, 219-222 (1989).

38. Poort, S. H. M., Meijerink, A. \& Blasse, G. Lifetime measurements in Eu² ${ }^{+}$-doped host lattices. J. Phys. Chem. Solids 58, 1451-1456 (1997).

39. Kato, K. \& Okamoto, F. Preparation and cathodoluminescence of CaS:Eu and $\mathrm{Ca}_{1-x} \mathrm{Sr}_{x} \mathrm{~S}:$ Eu phosphors. Jpn. J. Appl. Phys. 22, 76-78 (1983).

40. Joos, J. J., Meert, K. W., Parmentier, A. B., Poelman, D. \& Smet, P. F. Thermal quenching and luminescence lifetime of saturated green $\mathrm{Sr}_{1-x} \mathrm{Eu}_{x} \mathrm{Ga}_{2} \mathrm{~S}_{4}$ phosphors. Opt. Mater. 34, 1902-1907 (2012).

41. Zhang, $\mathrm{L}$. et al. The evolution and role of $\mathrm{NH}_{4} \mathrm{Cl}$ flux used to synthesize $\mathrm{Sr}_{2} \mathrm{SiO}_{4}: \mathrm{Dy}^{3+}$ phosphor by solid-state reaction method. J. Am. Ceram. Soc. 95 , 3871-3877 (2012)

42. Liu, L. H., Xie, R.-J., Zhang, C. N. \& Hirosaki, N. Role of fluxes in optimizing the optical properties of $\mathrm{Sr}_{0.95} \mathrm{Si}_{2} \mathrm{O}_{2} \mathrm{~N}_{2}: 0.05 \mathrm{Eu}^{2+}$ green-emitting phosphor. Materials 6, 2862-2872 (2013). 
43. Neefjes, I., Joos, J. J., Barandiarán, Z. \& Seijo, L. Mixed-valence lanthanideactivated phosphors: invariance of the intervalence charge transfer (IVCT) absorption onset across the series. J. Phys. Chem. C 124, 2619-2626 (2020).

44. Dexter, D. L. A theory of sensitized luminescence in solids. J. Chem. Phys. 21, 836-850 (1953).

45. Tagiev, O. B. \& Ganbarova, K. B. Energy transfer from $\mathrm{Tb}^{3+}$ to $\mathrm{Eu}^{2+}$ in $\mathrm{Ga}_{2} \mathrm{~S}_{3}$ : $\left(\mathrm{Eu}^{2+}, \mathrm{Tb}^{3+}\right)$ crystals. Semiconductors 49, 448-451 (2015).

46. Leyre, S. et al. Absolute determination of photoluminescence quantum efficiency using an integrating sphere setup. Rev. Sci. Instrum. 85, 123115 (2014).

47. Poelman, D., Doerschel, J. \& Smet, P. F. Cathodoluminescence study of the activator distribution in $\mathrm{SrS}: \mathrm{Cu}, \mathrm{Ag}$ thin luminescent films. Spectrochim. Acta B 59, 1775-1780 (2004).

48. Noto, L. L. et al. Photoluminescence and phase related cathodoluminescence dynamics of $\mathrm{Pr}^{3+}$ doped in a double phase of $\mathrm{ZnTa}_{2} \mathrm{O}_{6}$ and $\mathrm{ZnAl}_{2} \mathrm{O}_{4}$. Ceram. Int. 42, 5497-5503 (2016).

49. Martin, L. I. D. J., Poelman, D., Smet, P. F. \& Joos, J. J. Microscopic study of dopant distribution in europium doped $\mathrm{SrGa}_{2} \mathrm{~S}_{4}$ : impact on thermal quenching and phosphor performance. ECS J. Solid State Sci. Technol. 7, R3052-R3056 (2018).

50. Li, W. Y., Smet, P. F., Martin, L. I. D. J., Pritzel, C. \& Auf der Günne, J. S. Doping homogeneity in Co-doped materials investigated at different length scales. Phys. Chem. Chem. Phys. 22, 818-825 (2020).

51. Hirosaki, N., Takeda, T., Funahashi, S. \& Xie, R.-J. Discovery of new nitridosilicate phosphors for solid state lighting by the single-particlediagnosis approach. Chem. Mater. 26, 4280-4288 (2014).

52. Takeda, T., Hirosaki, N., Funahshi, S. \& Xie, R.-J. Narrow-band greenemitting phosphor $\mathrm{Ba}_{2} \mathrm{LiSi}_{7} \mathrm{AlN}_{12}: \mathrm{Eu}^{2+}$ with high thermal stability discovered by a single particle diagnosis approach. Chem. Mater. 27, 5892-5898 (2015).

53. Wang, X. J., Funahashi, S., Takeda, T., Suehiro, T., Hirosaki, N. \& Xie, R. J. Structure and luminescence of a novel orange-yellow-emitting $\mathrm{Ca}_{1.62} \mathrm{Eu}_{0.38} \mathrm{Si}_{5} \mathrm{O}_{3} \mathrm{~N}_{6}$ phosphor for warm white LEDs, discovered by a singleparticle-diagnosis approach. J. Mater. Chem. C 4, 9968-9975 (2016).

54. Packwood, R. H. \& Brown, J. D. A gaussian expression to describe $\phi(\rho z)$ curves for quantitative electron probe microanalysis. X-Ray Spectrom. 10 138-146 (1981).

55. Smet, P. F., Botterman, J., Parmentier, A. B. \& Poelman, D. Thermal quenching at the microscopic level in multi-phase thiosilicate phosphors. Opt. Mater. 35, 1970-1975 (2013).

56. Bettinelli, M., Speghini, A., Piccinelli, F., Ueda, J. \& Tanabe, S. Energy transfer processes in $\mathrm{Sr}_{3} \mathrm{~Tb}_{0.90} \mathrm{Eu}_{0.10}\left(\mathrm{PO}_{4}\right)_{3}$. Opt. Mater. 33, 119-122 (2010).

57. Mott, N. F. On the absorption of light by crystals. Proc. R. Soc. Lond. Ser. A 167, 384-391 (1938).

58. Ando, M. \& Ono, Y. A. Temperature effects in the emission characteristics of CaS:Eu thin-film electroluminescent devices. J. Cryst. Growth 117, 969-974 (1992).

59. Nyenge, R. L. et al. Thermal quenching, cathodoluminescence and thermoluminescence study of $\mathrm{Eu}^{2+}$ doped CaS powder. J. Alloy. Compd. 657, 787-793 (2016).

60. Meyer, B., Phillips, L. F. \& Smith, J. J. Temperature dependence of intersystem crossing-lifetime and intensity of $\mathrm{SO}_{2}$ phosphorescence in low-temperature solids. Proc. Natl Acad. Sci. USA 61, 7-11 (1968).

61. Stacy, W. T. \& Swenberg, C. E. Temperature dependence of intersystem crossing in crystalline anthracene. J. Chem. Phys. 52, 1962-1965 (1970).

62. Yang, L., Zhang, N., Zhang, R. Y., Wen, B., Li, H. L. \& Bian, X. B. A CaS:Eu based red-emitting phosphor with significantly improved thermal quenching resistance for LED lighting applications. Mater. Lett. 129, 134-136 (2014).

63. Struck, C. W. \& Fonger, W. H. Thermal quenching of $\mathrm{Tb}^{3+}, \mathrm{Tm}^{3+}, \mathrm{Pr}^{3+}$, and $\mathrm{Dy}^{3+} 4 \mathrm{f}^{n}$ emitting states in $\mathrm{La}_{2} \mathrm{O}_{2}$ S. J. Appl. Phys. 42, 4515-4516 (1971).

64. Struck, C. W. \& Fonger, W. H. Unified model of temperature quenching of narrow-line and broad-band emissions. J. Lumin. 10, 1-30 (1975).

65. Struck, C. W. \& Fonger, W. H. Temperature quenching of luminescence for linear and derivative nuclear operators. J. Lumin. 14, 253-279 (1976).

66. Koepke, C., Lempicki, A. \& Wojtowicz, A. J. Luminescence quenching of strongly coupled systems. Phys. Stat. Sol. B 179, 233-240 (1993).

67. Barandiarán, Z., Meijerink, A. \& Seijo, L. Configuration coordinate energy level diagrams of intervalence and metal-to-metal charge transfer states of dopant pairs in solids. Phys. Chem. Chem. Phys. 17, 19874-19884 (2015).

68. MacKeen, $\mathrm{C}$. et al. The complexity of the $\mathrm{CaF}_{2}: \mathrm{Yb}$ system: a huge, reversible, X-ray-induced valence reduction. J. Phys. Chem. C 121, 28435-28442 (2017).

69. Barandiarán, Z., Bettinelli, M. \& Seijo, L. Color control of $\mathrm{Pr}^{3+}$ luminescence by electron-hole recombination energy transfer in $\mathrm{CaTiO}_{3}$ and $\mathrm{CaZrO}_{3}$. J. Phys. Chem. Lett. 8, 3095-3100 (2017).

70. Parmentier, A. B., Joos, J. J., Smet, P. F. \& Poelman, D. Luminescence of Ytterbium in CaS and SrS. J. Lumin. 154, 445-451 (2014).

71. Pust, P. et al. Narrow-band red-emitting $\operatorname{Sr}\left[\mathrm{LiAl}_{3} \mathrm{~N}_{4}\right]: \mathrm{Eu}^{2+}$ as a nextgeneration LED-phosphor material. Nat. Mater. 13, 891-896 (2014).
72. Suta, M. \& Wickleder, C. Spin crossover of $\mathrm{Yb}^{2+}$ in $\mathrm{CsCaX}_{3}$ and $\mathrm{CsSrX}_{3}(\mathrm{X}=$ $\mathrm{Cl}, \mathrm{Br}, \mathrm{I})-$ a guideline to novel halide-based scintillators. Adv. Funct. Mater. 27, 1602783 (2017)

73. Suta, M., Urland, W., Daul, C. \& Wickleder, C. Photoluminescence properties of $\mathrm{Yb}^{2+}$ ions doped in the perovskites $\mathrm{CsCaX}_{3}$ and $\mathrm{CsSrX}_{3}(\mathrm{X}=\mathrm{Cl}$, $\mathrm{Br}$, and I) - a comparative study. Phys. Chem. Chem. Phys. 18, 13196-13208 (2016).

74. Dorenbos, P. A review on how lanthanide impurity levels change with chemistry and structure of inorganic compounds. ECS J. Solid State Sci. Technol. 2, R3001-R3011 (2013).

75. Joos, J. J., Poelman, D. \& Smet, P. F. Energy level modeling of lanthanide materials: review and uncertainty analysis. Phys. Chem. Chem. Phys. 17, 19058-19078 (2015).

76. Karlström, G. et al. MOLCAS: a program package for computational chemistry. Comput. Mater. Sci. 28, 222-239 (2003).

77. Douglas, M. \& Kroll, N. M. Quantum electrodynamical corrections to the fine structure of helium. Ann. Phys. (N.Y.) 82, 89-155 (1974).

78. Hess, B. A. Relativistic electronic-structure calculations employing a twocomponent no-pair formalism with external-field projection operators. Phys. Rev. A 33, 3742-3748 (1986).

79. Roos, B. O., Lindh, R., Malmqvist, P. Å, Veryazov, V. \& Widmark, P. O. Main group atoms and dimers studied with a new relativistic ANO basis set. J. Phys. Chem. A 108, 2851-2858 (2004).

80. Roos, B. O., Lindh, R., Malmqvist, P. Å, Veryazov, V., Widmark, P. O. \& Borin, A. C. New relativistic atomic natural orbital basis sets for lanthanide atoms with applications to the Ce diatom and $\mathrm{LuF}_{3}$.J. Phys. Chem. A 112, 11431-11435 (2008)

81. Roos, B. O., Taylor, P. R. \& Siegbahn, P. E. M. A complete active space SCF method (CASSCF) using a density-matrix formulated super-CI approach. Chem. Phys. 48, 157-173 (1980).

82. Siegbahn, P. E. M., Heiberg, A., Roos, B. O. \& Levy, B. Comparison of the super-CI and the Newton-Raphson scheme in the complete active space SCF method. Phys. Scr. 21, 323-327 (1980).

83. Siegbahn, P. E. M., Heiberg, A., Almlöf, J. \& Roos, B. O. The complete active space SCF (CASSCF) method in a Newton-Raphson formulation with application to the HNO molecule. J. Chem. Phys. 74, 2384-2396 (1981).

84. Barandiarán, Z. \& Seijo, L. Radial correlation effects on interconfigurational excitations at the end of the lanthanide series: a restricted active space second order perturbation study of $\mathrm{Yb}^{2+}$ and $\mathrm{SrCl}_{2}: \mathrm{Yb}^{2+}$. J. Chem. Phys. 138, 074102 (2013).

85. Andersson, K., Malmqvist, P.-Å, Roos, B. O., Sadlej, A. J. \& Wolinski, K. Second-order perturbation theory with a CASSCF reference function. J. Phys. Chem. 94, 5483-5488 (1990).

86. Andersson, K., Malmqvist, P.-Å \& Roos, B. O. Second-order perturbation theory with a complete active space self-consistent field reference function. $J$. Chem. Phys. 96, 1218-1226 (1992).

87. Zaitsevskii, A. \& Malrieu, J.-P. Multi-partitioning quasidegenerate perturbation theory. A new approach to multireference Møller-Plesset perturbation theory. Chem. Phys. Lett. 233, 597-604 (1995).

88. Finley, J., Malmqvist, P.-Å, Roos, B. O. \& Serrano-Andrés, L. The multi-state CASPT2 method. Chem. Phys. Lett. 288, 299-306 (1998).

89. Ghigo, G., Roos, B. O. \& Malmqvist, P.-A A modified definition of the zerothorder Hamiltonian in multiconfigurational perturbation theory (CASPT2). Chem. Phys. Lett. 396, 142 (2004).

90. Hess, B. A., Marian, C. M., Wahlgren, U. \& Gropen, O. A mean-field spin-orbit method applicable to correlated wavefunctions. Chem. Phys. Lett. 251, 365-371 (1996).

91. Malmqvist, P.-Å, Roos, B. O. \& Schimmelpfennig, B. The RASSI approach with spin-orbit coupling. Chem. Phys. Lett. 357, 230-240 (2002).

92. Paulovic, J., Nakajima, T., Hirao, K., Lindh, R. \& Malmqvist, P.-A. Relativistic and correlated calculations on the ground and excited states of ThO. J. Chem. Phys. 119, 798-805 (2003).

93. Barandiarán, Z. \& Seijo, L. The ab initio model potential representation of the crystalline environment. Theoretical study of the local distortion on $\mathrm{NaCl}$ : $\mathrm{Cu}^{+}$. J. Chem. Phys. 89, 5739-5746 (1988).

94. Seijo, L. \& Barandiarán, Z. Ab initio model potential study of local distortions around $\mathrm{Cr}^{+}$and $\mathrm{Cr}^{3+}$. J. Chem. Phys. 94, 8158 (1991).

\section{Acknowledgements}

J.J.J. and D.V.d.H. acknowledge the UGent Special Research Fund (BOF/PDO/2017/ 002101 and BOF16/DOC/327). This work was partially supported by Ministerio de Economía y Competitividad, Spain (Dirección General de Investigación y Gestión del Plan Nacional de I + D + i, MAT2017-83553-P). We acknowledge the Fund for Scientific Research-Flanders (FWO) for financial support (project I002418N) and a travel grant 
(V416818N) and the European Synchrotron Radiation Facility (ESRF) for the allocation of beamtime (beamline ID26).

\section{Author contributions}

J.J.J.: Conceptualization, methodology, software, validation, formal analysis, investigation, data curation, writing original draft, writing review and editing, visualization, supervision, project administration, and funding acquisition. D.V.d.H.: Methodology, validation, investigation, writing review and editing. L.I.D.J.M.: Investigation, writing review and editing. L.A.: Investigation, writing review and editing. P.F.S.: Methodology, resources, data curation, writing review and editing, supervision, funding acquisition. Z.B.: Methodology, software, resources, data curation, writing review and editing, supervision, funding acquisition. L.S.: Methodology, software, resources, data curation, writing review and editing, supervision, funding acquisition.

\section{Competing interests}

The authors declare no competing interests.

\section{Additional information}

Supplementary information is available for this paper at https://doi.org/10.1038/s41467020-17469-x.

Correspondence and requests for materials should be addressed to J.J.J.
Peer review information Nature Communications thanks Eszter Borbas, and other, anonymous, reviewers for their contribution to the peer review of this work.

Reprints and permission information is available at http://www.nature.com/reprints

Publisher's note Springer Nature remains neutral with regard to jurisdictional claims in published maps and institutional affiliations.

\section{(c) (i)}

Open Access This article is licensed under a Creative Commons Attribution 4.0 International License, which permits use, sharing, adaptation, distribution and reproduction in any medium or format, as long as you give appropriate credit to the original author(s) and the source, provide a link to the Creative Commons license, and indicate if changes were made. The images or other third party material in this article are included in the article's Creative Commons license, unless indicated otherwise in a credit line to the material. If material is not included in the article's Creative Commons license and your intended use is not permitted by statutory regulation or exceeds the permitted use, you will need to obtain permission directly from the copyright holder. To view a copy of this license, visit http://creativecommons.org/ licenses/by/4.0/.

(c) The Author(s) 2020 